\title{
Morfotipo del futbolista profesional de la Comunidad Autónoma de Madrid. Composición corporal
}

\author{
A.. ${ }^{1}$ HERRERO DE LUCAS, M. D. ${ }^{1}$ CABANAS ARMESILLA, I. ${ }^{2}$ MAESTRE LÓPEZ \\ ${ }^{1}$ Departamento de Anatomía y Embriología Humana II, \\ Facultad de Medicina, Universidad Complutense de Madrid, España \\ ${ }^{2}$ Grupo de investigación de nutrición de las poblaciones humanas en salud y enfermedad, Facul- \\ tad de Medicina, Universidad Complutense de Madrid, España
}

\begin{abstract}
Resumen
La composición corporal es dentro del estudio cineantropométrico de la población de atletas la que se utiliza con mayor frecuencia, ya que permite de manera inmediata tener una visión global del morfotipo del futbolista sobre el cual se puede actuar modificándolo fácilmente por medio de una adecuada preparación física. Se estudiaron 56 jugadores de fútbol profesional que desarrollan su actividad física en la Comunidad Autónoma de Madrid, de manera voluntaria, obteniéndose el consentimiento informado previo de cada uno de ellos de acuerdo a la legislación vigente. La composición corporal se realizó mediante la segmentación del cuerpo humano en cuatro componentes. El análisis estadístico se realizó utilizando el programa de software compatible con PC SPSS 11.5. La edad media de los jugadores estudiados es de 26.087 (edad decimal) \pm 3.48 años, la talla media es de $177.87 \pm 6.78 \mathrm{~cm}$., el peso medio es de $77.24 \pm 5.60 \mathrm{Kg}$., el porcentaje de grasa medio es de un 10.42 $\pm 0.70 \%$, el porcentaje muscular es de un 50,04 $\pm 0.79 \%$, el porcentaje óseo es de un $15.44 \pm 0.73 \%$ y el peso residual de un $24.10 \%$.
\end{abstract}

Palabras clave: Cineantropometría, Composición Corporal, Morfotipo, Fútbol

\begin{abstract}
Body composition is the mostly used of kinanthropometric parameters in evaluating athletic population because enhances an immediate overall morphotype of the football player modifying their constitution promptly by an appropriate physical training. 56 football players performing in teams of the Autonomous Community of Madrid were studied, voluntarily, obtaining a previous informed consent as established by local legislation. Body composition was divided in four body segments. Statistical analysis realised by PC compatible software SPSS 11.5. Mean age of the players studied was 26.087 (decimal age \pm 3.48 years, mean height of $177.87 \pm 6.78 \mathrm{~cm}$., mean weight of $77.24 \pm 5.60 \mathrm{Kg}$., mean fat percentage of $10.42 \pm 0.70 \%$, mean muscular percentage of 50,04 $\pm 0.79 \%$, mean bone percentage of $15.44 \pm 0.73 \%$ and residual weight of $24.10 \%$.
\end{abstract}

Key words: Kinanthropometry, Body Composition, Morphotype, Football, Soccer

\section{Introducción}

La Composición corporal sólo la podemos definir en la conjunción de los significantes etimológicos de ambos términos y en el sentido que

Correspondencia:

Dr. Angel Herrero de Lucas.

Departamento de Anatomía y Embriología Humana II, Facultad de Medicina, Universidad Complutense de Madrid. c/ Plaza Ramón y Cajal s/n. 28040 Madrid. Tfno: 6309887 50. Fax: 916104934.

E-mail: herrero_angel@hotmail.com nos indica la última edición vigente del Diccionario de la Lengua Española de la Real Academia Española[1], utilizando sólo aquellas acepciones relacionadas con el ámbito científico, como: Composición, del latín compositio, -ônis; 1 . Acción y efecto de componer. 3. Compostura, circunspección.4. Obra científica, literaria o musical y Corporal, del latín corporâlis; adj."Perteneciente o relativo al cuerpo, especialmente al humano". Si bien otros diccionarios lo han definido en su conjunto, considerando la composición corporal como las cantidades relativas de músculo, hueso y grasa corporal. La composición corporal suele estar dividida en grasa (por- 
centaje de grasa corporal) y masa magra (porcentaje de masa magra corporal) [2]. El estudio de la composición corporal no floreció entre los biólogos porque las masas de tejidos de los compartimentos corporales parecían muy elementales, ignorando los taxonomistas la grasa por su obvia relación con el estado nutricional; además, los biólogos relacionados con la morfogénesis negaron las medidas tisulares por ser anatómicamente muy groseras para explicar las variaciones en el crecimiento y la forma. El paso del tiempo cambió la mentalidad pretérita, y donde antes no se daba importancia a la composición corporal por las razones aducidas, hoy día ocurre todo lo contrario debido a la relación de los diferentes tejidos con diversas patologías que preocupan a la comunidad científica y a la población en general. El componente graso es directamente proporcional a la mortandad del hombre, sobre todo por su relación con enfermedades cardiovasculares, metabólicas,...; el componente muscular consigue relevancia por el creciente interés de las sociedades por una dietética correcta donde la importancia de las proteínas en la misma es cada vez mayor, añadido a su relación con el índice de crecimiento muscular; y finalmente el componente óseo del cuerpo humano por el problema que la osteoporosis ha adquirido en la actualidad.

El aspecto de la valoración fisiológica en el mundo del fútbol ha experimentado un auge considerable en los últimos años, de tal forma que actualmente se seleccionan jugadores de fútbol en base no solo a su calidad técnica sino también y cada vez en mayor medida a su capacidad física. La composición corporal específicamente en el ámbito deportivo es ampliamente utilizada por ofrecer valores del morfotipo que se pueden modificar fácilmente mediante la dieta o la preparación física con el fin de obtener un mayor rendimiento físico de los atletas considerados.

El objetivo de este estudio es analizar las características antropométricas específicas de una población de futbolistas que desarrollan su actividad física en equipos de la Comunidad Autónoma de Madrid para establecer su patrón cineantropométrico específico que los identifique con el fin de obtener futuros patrones de referencia de la población española en su conjunto.

\section{Materiales y Métodos}

Se estudiaron 56 jugadores de fútbol pertenecientes al C.D. Leganés, S.A.D., equipo profesional de la Comunidad Autónoma de Madrid. En el estudio se incluyeron aquellos futbolistas nacidos fuera de la Comunidad Autónoma de Madrid o no nacionales por el carácter pluriétnico y supranacional que han adquirido las grandes metrópolis en general y el fútbol en particular, donde salvo contadas excepciones la multiplicidad es el factor dominante.

Las medidas se realizaron siguiendo las normas y técnicas de medición del G.R.E.C.-I.W.G.K. (Grupo Español de Cineantropometría-International Working Group in Kinanthropometry)[3,4,5]. Se obtuvieron los correspondientes consentimientos informados de acuerdo a la legislación vigente y el tratamiento de la información estuvo de acuerdo a los parámetros establecidos en la ley de protección de datos.

Se realizaron 38 medidas antropométricas: peso; talla; alturas: acromial, radial, ileoespinal, trocantérea y tibial; longitudes: brazo, antebrazo, mano, muslo, pierna y pie; pliegues cutáneos: subescapular, suprailíaco, abdominal, tríceps, muslo anterior y medial pierna; diámetros: biacromial, transverso y antero-posterior de tórax, biileocrestal, biepicondíleo húmero, biestiloideo, bicondíleo fémur y bimaleolar; y perímetros: torácico-axilar, cintura, cadera, brazo relajado, brazo contraído, antebrazo, muñeca, muslo superior, muslo inferior, pierna y tobillo. Los instrumentos de medida utilizados fueron los propios de la maleta de cineantropometría homologados, además de una báscula (Soehnle) y un plicómetro Holtain.

Se marcaron los puntos anatómicos de referencia en el lado derecho del cuerpo previo a la obtención de medidas.

La metodología seguida es la propuesta por Heath y Carter[6,7,8], con un fraccionamiento de la composición corporal en cuatro componentes si-

Tabla 1. Muestra poblacional

\begin{tabular}{|c|c|c|c|}
\hline & Edad Decimal (años) & Talla (cm.) & Peso (Kg.) \\
\hline media & 26.087 & 177.87 & 77.224 \\
\hline DE & 3.48 & 6.78 & 5.60 \\
\hline
\end{tabular}


guiendo la estrategia de De Rose y Guimaraes[9], utilizando para el cálculo del peso graso la fórmula de Faulkner[10], para el peso óseo la de Rocha[11], para el peso muscular la de Matiegka[12] y para el peso residual la de Würch[13].

El análisis estadístico se llevó a cabo por medio del programa de software para PC, SPSS 11.5 utilizándose la t Student para muestras relacionadas y el análisis de la varianza (ANOVA) para la comparación de medias, ambas con un nivel de significación del 95\% ( $\mathrm{p}<0.05)$.

\section{Resultados}

Las población estudiada la conforman 56 jugadores de fútbol divididos en 5 porteros, 15 defensas, 21 centrocampistas y 15 delanteros; del conjunto de todos ellos el $87.04 \%$ son diestros, el $11.11 \%$ zurdos y el 1.85 ambidextros.

Los porteros son los jugadores de fútbol de mayor edad, mayor talla y mayor peso.

\section{Composición Corporal}

El perfil lipídico basado en la distribución de pliegues cutáneos y el perfil óseo basado en la longitud de diámetros se muestran en la tabla 2. La distribución de lípidos se presenta en forma de sumatorio de 4 pliegues (pliegue cutáneo subescapular, suprailíaco, abdominal y tríceps). El perfil óseo considera para su cálculo los diámetros biestiloideo y bicondíleo de fémur.

Los porteros son los jugadores de fútbol con mayor perfil lipídico, al ser los que presentan un mayor sumatorio de 4 pliegues cutáneos. No ocu- rre lo mismo al analizar el perfil óseo, donde encontramos que aquellos futbolistas que presentan el mayor diámetro biestiloideo son los porteros, sin embargo llama la atención que en el diámetro bicondíleo de fémur no se corresponden los datos hallados con los hasta el momento presentados, al ser los deportistas que presentan una longitud superior de dicha medida los defensas, y así difieren al obtener un resultado más grande que el de los porteros.

El fraccionamiento de la composición corporal en cuatro componentes es posible de acuerdo a las fórmulas propias de cada uno de ellos y establecidas previamente; para lo cual utilizamos los datos obtenidos y mostramos aquellos segmentos del morfotipo del futbolista sobre el cual podemos actuar para mejorar su rendimiento deportivo, tal como se puede apreciar en la tabla 3.

Los porteros son los jugadores de fútbol con un mayor porcentaje de grasa corporal y masa muscular. Resaltamos como al analizar el porcentaje óseo, en los porteros no solo no se obtiene el valor más alto, sino que ocurre todo lo contrario, siendo el tanto por ciento más bajo.

\section{Discusión}

El perfil lipídico y óseo encontrado en la literatura muestra valores de $31.5 \mathrm{~mm}$. y $28.2 \mathrm{~mm}$. en el sumatorio de 4 pliegues para jugadores de fútbol de Puerto Rico[14] y Bahrain[15] respectivamente; mientras que el obtenido en nuestro estudio se encuentra en medio de ambos valores, lo que contrasta on los datos de los futbolistas puertorriqueños, induciéndonos a pensar que se utilizó una fór-

Tabla 2. Perfil lipídico y óseo

\begin{tabular}{|c|c|c|c|}
\hline & $\begin{array}{c}\text { Sumatorio 4 Pliegues } \\
(\mathbf{m m} .)\end{array}$ & $\begin{array}{c}\text { Diámetro Biestiloideo } \\
(\mathbf{c m} .)\end{array}$ & $\begin{array}{c}\text { Diámetro Bicondíleo Fémur } \\
(\mathbf{c m} .)\end{array}$ \\
\hline media & 30.28 & 5.60 & 9.61 \\
\hline DE & 4.33 & 0.32 & 0.37 \\
\hline
\end{tabular}

Tabla 3. Composición corporal

\begin{tabular}{|c|c|c|c|}
\hline & \% Grasa & \% Muscular & \% Oseo \\
\hline media & 10.42 & 50.04 & 15.44 \\
\hline DE & 0.70 & 0.79 & 0.73 \\
\hline
\end{tabular}


mula diferente a la de Faulkner, ya que se trata de países con muy poca tradición futbolística, sin poder descartar que se deba a las características genéticas de la población del país caribeño.

El diámetro biestiloideo de futbolistas españoles de élite es de $5.8 \mathrm{~cm}[16]$. El deporte que comparte con el fútbol la mayor similitud técnico - táctica es el fútbol sala; y en éste encontramos un diámetro biestiloideo de $5.39 \mathrm{~cm}$.[17] en jugadores profesionales. Comparando los diferentes juegos que reciben denominación de fútbol, incluyendo en esta categoría el rugby puesto que se considera el padre del fútbol o más bien éste una variación del anterior, los diámetros medios en jugadores de rugby perteneciente a la primera división nacional española es de $5.7 \mathrm{~cm}[18]$. Comparamos fútbol con atletismo porque los futbolistas también entrenan en gran medida la carrera continua, pese a las diferencias técnicas que existen entre ambos deportes; encontrando que los diámetros biestiloideo medios en corredores de maratón tienen una longitud de $5.82 \mathrm{~cm}[19]$. En todos los casos el diámetro hallado en nuestro estudio es inferior a los encontrados en la bibliografía internacional, y como cabría esperar los valores son muy próximos a los futbolistas de élite españoles, siendo parejos asimismo a los jugadores de rugby, alejándose de los corredores de fondo y lo que es más sorprendente, de los jugadores de fútbol-sala, sin que halla una explicación razonable para este último hallazgo.

El diámetro bicondíleo de fémur de futbolistas españoles de élite es de $9.8 \mathrm{~cm}$ [16]. El deporte que comparte con el fútbol la mayor similitud técnico táctica es el fútbol sala; y en éste encontramos un diámetro bicondíleo de fémur de $9.44 \mathrm{~cm}$.[17] en jugadores profesionales. Comparando los diferentes juegos que reciben denominación de fútbol, incluyendo en esta categoría el rugby puesto que se considera el padre del fútbol o más bien éste una variación del anterior, los diámetros medios en jugadores de rugby perteneciente a la primera división nacional española es de $9 \mathrm{~cm}$ [18]. Los diámetros medios en futbolistas americanos son de 10.5 $\mathrm{cm}$.[20] Comparamos fútbol con atletismo porque los futbolistas también entrenan en gran medida la carrera continua, pese a las diferencias técnicas que existen entre ambos deportes; encontrando que los diámetros bicondíleos de fémur medios en corredores de maratón tienen una longitud de 8.89 cm.[19] Los resultados de este estudio, a diferencia del otro diámetro muestra unos valores intermedios entre el fútbol y el fútbol-sala, alejándose mucho por defecto del fútbol americano, como se- ría lógico, y por exceso de los corredores de fondo, como cabría esperar, pero también de los jugadores de rugby, este ya un dato sorprendente, sin explicación aparente.

El porcentaje de grasa de futbolistas españoles de élite es de $11.16 \%$ [16], siendo para otros países; futbolistas sudamericanos participantes en la Copa América es del 11\%[21]; en italianos es del $11.56 \%$ [22]; $11.2 \%$ en jugadores de la primera división inglesa[23]; $11.8 \%$ en holandeses[24]; $11.0 \%$ en portugueses[25], los cuales son futbolistas mediterráneos que además aceptan a los futbolistas brasileños como oriundos. Existe un estudio que analizó 248 futbolistas de la primera división de México (población formada por 198 mexicanos y 50 de Brasil y Argentina) entre 1973 y 2000, cuyos resultados se presentan por décadas (70's, 80's y 90’s); la media de éstos en la primera década estudiada es de $15 \%, 12 \%$ en la segunda y $10 \%$ en la tercera[26]. 9.59\% en futbolistas de Estados Unidos[27] pertenecientes a la N.A.S.L. (North American Soccer League); $12.8 \%$ en la República Dominicana[28], 10.8\% en futbolistas australianos de la selección nacional[29]; 12.4\% en la selección nacional finlandesa[30]; $12.3 \%$ en la selección de Arabia Saudita[31]. El deporte que comparte con el fútbol la mayor similitud técnico - táctica es el fútbol sala[17]; y en éste encontramos un porcentaje graso de $7 \%$ en jugadores profesionales, valor lo suficientemente bajo para considerar que se ha obtenido con una fórmula diferente a la de Faulkner. Comparando los diferentes juegos que reciben denominación de fútbol, incluyendo en esta categoría el rugby puesto que se considera el padre del fútbol o más bien éste una variación del anterior, los porcentajes medios en jugadores de rugby perteneciente a la primera división nacional española es de $10.47 \%$ [18]. Los porcentajes medios en futbolistas americanos son de $13.8 \%$ [20]. Comparamos fútbol con atletismo porque los futbolistas también entrenan en gran medida la carrera continua, pese a las diferencias técnicas que existen entre ambos deportes; encontrando que los porcentajes de grasa medios en corredores de maratón tienen una longitud de $8.2 \%[19]$. Los resultados de la composición corporal son los más paradójicos, ya que muestran una dispersión por defecto frente a los países de mayor tradición futbolística (españoles de élite, holandeses, ingleses, etc.), siendo próximos a países de tradición futbolística mediterránea como Portugal o futbolistas sudamericanos de élite, sin que haya predominancia de jugadores latinoamericanos en nuestro estudio, y por tanto sorprende la 
mayor similitud de los mismos frente a éstos últimos que a los que representan los jugadores españoles más capacitados. Los resultados próximos de naciones poco competitivas a nivel internacional nos sugieren que se calcularon con fórmulas diferentes a la de Faulkner.

En la bibliografía internacional es difícil encontrar porcentajes musculares, óseos o residual, ya que la mayoría de los artículos expresan estos resultados en $\mathrm{Kg}$. de peso de cada uno de los componentes.

El porcentaje muscular de futbolistas españoles de élite es de 52.2\%[16], siendo para otros países; $47.3 \%$ en jugadores ingleses[32]. Comparamos fútbol con atletismo porque los futbolistas también entrenan en gran medida la carrera continua, pese a las diferencias técnicas que existen entre ambos deportes; encontrando que los porcentajes musculares medios en corredores de fondo tienen una longitud de 47.2\%[33]. El resultado de nuestro estudio muestra unos valores intermedios entre el fútbol anglosajón y el español, donde cabe destacar el mayor desarrollo muscular de los futbolistas hispanos, en disonancia con la creencia general de un mayor desarrollo físico de los países del norte de Europa. Es normal la distancia por exceso frente a los corredores de fondo.

El porcentaje óseo de futbolistas españoles de élite es de 16\%[16]. Comparamos fútbol con atletismo porque los futbolistas también entrenan en gran medida la carrera continua, pese a las diferencias técnicas que existen entre ambos deportes; encontrando que los porcentajes óseos medios en corredores de fondo tienen una longitud de $18.38 \%[33]$. Los datos obtenidos en nuestro estudio muestran la lógica de valores muy próximos a los futbolistas de élite españoles, alejándose por exceso de los corredores de fondo.

El porcentaje residual de futbolistas españoles de élite es de 24\%[16]. Comparamos fútbol con atletismo porque los futbolistas también entrenan en gran medida la carrera continua, pese a las diferencias técnicas que existen entre ambos deportes; encontrando que los porcentajes residuales medios en corredores de fondo tienen una longitud de $27.92 \%$ [33]. Los valores hallados coinciden con los futbolistas de élite nacionales y se distancian de los corredores de fondo.

\section{Conclusiones}

La importancia de la composición corporal en el deporte en general y el fútbol en particular es la posibilidad de mejorar el rendimiento deportivo fácilmente actuando sobre parámetros muy específi$\cos$. En este sentido podemos modificar el porcentaje graso con una dieta adecuada del futbolista, eliminando un peso superfluo no útil, y el porcentaje muscular con un adecuado programa de preparación física bien en ampo, gimnasio o ambos al mismo tiempo. Si bien el estudio se centra en los jugadores de fútbol de élite, éstas premisas se pueden aplicar a los deportistas semiprofesionales o aficionados, e incluso a los futbolistas jóvenes de cualquier edad, en este último caso introduciendo un nuevo parámetro a valorar cual sería el percentil de crecimiento.

Otro aspecto de gran importancia a valorar es la biomecánica específica del fútbol, al ser un deporte que conjuga varios gestos técnicos bien en solitario o conjugados (carrera lenta, carrera rápida, salto, giro, etc.). La modificación de la biomecánica del futbolista como atleta individualizado, en función de su composición corporal actual o de la misma objetivo final, reduciría la lesionabilidad del deportista en gestos técnicos muy forzados, por el hecho de que una adecuada composición corporal se acompaña de una mayor fuerza por aumento de masa corporal y un menor peso por mejora del porcentaje graso, beneficioso para realizar los mismos.

\section{Bibliografía}

1. En: Real Academia Española, editors. Diccionario de la Lengua Española, 22ª Ed. Madrid: 2001.

2. En: Paidotribo, editors. Diccionario Paidotribo de la Actividad Física y el Deporte. Barcelona: 1999.

3. En: FEMEDE, editors. Manual de Cineantropometría. Murcia: 1993.

4. Ross WD, Brown SR, Hebbelinck M, Faulkner RA. Kinanthropometry, terminology and landmarks. En: Shepard RJ, Lavallee H, editors. Physical fitness assessment. Springfield: 1978. p. 44-50.

5. Ross WD, Marfell-Jones MJ. Kinanthropometry. En: MacDougall JD, Wenger HA, Grenn HJ, editors. Physiological testing of the high-performance athlete. Illinois: 1991. p. 223-308.

6. En: San Diego State University, editors. The HeathCarter somatotype method. San Diego: 1975

7. Carter JEL, Heath BH. Sport and physical performance. En: Lasker GW, Mascie-Taylor CGN, Roberts DF, editors. Somatotyping, development and applications. New York: 1990. p. 198-290.

8. Carter JEL, Ross WD, Duquet W, Aubry SP. Advances in somatotype methodology and analysis. Yearb Phys Anthropol 1983; 26; 193-213.

9. De Rose EH, Guimaraes ACA. Model for optimization of somatotype in young athletes. En: 
Ostyn M, Bremen G, Simons J, editors. Kinanthropometry II. Baltimore: 1980. p. 222.

10. Faulkner JA. Physiology of swimming and diving. En: Falls HS, editors. Exercise physiology. Baltimore: 1968. p. 417.

11. Rocha MSL. Peso óseo do brasileiro de ambos os sexos de 17 a 25 anhos. Arquivos Anatomía e Antropología 1975; 1; 445-451.

12. Matiegka J. The testing of physical efficiency. Am S Phys Anthrop 1922; 4; 223-230.

13. Würch A. Le femme et le sport. Med Sport Francaise 1974; 4; 1.

14. Rivera MA, Avella FA. Características antropométricas y fisiológicas de futbolistas puertorriqueños. Arch Med Deporte 1992; 9; 35; 265 277.

15. Musaiger AO, Ragheb MA, Al-Marzooq G. Body composition of athletes in Bahrain. Brit J Sports Med 1994; 28; 157-159.

16. Casajús JA, Aragonés MT. Estudio cineantropométrico del futbolista profesional español. Arch Med Deporte 1997; 14; 59; 177-184.

17. En: Fundación Real Madrid, editors. Estudio de los diámetros en jugadores amateurs y profesionales de fútbol-sala. Congreso Internacional del Fútbol y las Ciencias del Deporte, 2000.

18. Urraca JM, Martínez J, Montoliú MA, Maestro A, Méndez D, Guerediaga X, Del Valle $M$. Estudio comparativo del somatotipo en jugadores de rugby. Arch Med Deporte 1999; 16, 69; 39-45.

19. Ruíz C, Galiano D, Gutiérrez JA. Modificaciones de la composición corporal durante la carrera de maratón. Arch Med Deporte 1971; 2; 5; 35-41.

20. Smith JF, Mansfield ER. Body composition prediction in university football players. Med Sci Sports Exerc 1984; 16; 4; 398-405.

21. Rienzi E, Drust B, Reilly T, Carter JEL, Martin A. Investigation of anthropometric and work-rate profiles of elite South American international soccer players. J Sports Med Phys Fitness 2000; 40; 2; 162 169.

22. Gualdi-Russo E, Gruppioni G, Gueresi P, Belcastro MG, Marchesini V. Skinfolds and body composition of sports participants. J Sports Med
Phys Fitness 1992; 32; 3; 303-313.

23. Strudwick A, Reilly T, Doran D. Anthropometric and fitness profiles of elite players in two football codes. J Sports Med Phys Fitness 2002; 42; 2; 239242.

24. Vos JA. Physiological comparison between Dutch soccer players and other teamsport men. En: Vechiet $\mathrm{L}$, editors. Proceedings $1^{\text {st }}$ international congress on sports medicine applied to football. Rome: 1980. p. 695-701.

25. Puga N, Ramos J, Agostinho J, Lomba I, Costa O, Falcäo F. Physical profile of a portuguese first división profesional football team. J Sports Sci 1991; 9; 150-151.

26. Díaz FJ, Montano JG, Melchor MT, García MR, Guerrero JH, Rivera AE, Tovar JA, Moreno MF. Changes of physical and functional characteristics in soccer players. Rev Invest Clin 2003; 55; 5; 528534.

27. Raven P, Gettman L, Pollock M, Cooper K. A physiological evaluation of profesional soccer players. Brit J Sports Med 1976; 10; 4; 209-216.

28. En: Comité Olímpico Dominicano, editors. Estudio de la composición corporal y somatotipo de atletas dominicanos. Santo Domingo: 1987.

29. Kirkendall DT. The applied sport science of soccer. Phys Sportsmed 1985; 13; 53-59.

30. Rahkila P, Luhtanen P. Physical fitness profile of finnish national soccer teams candidates. Science and Football 1991; 5; 30-34.

31. Al-Hazzaa HM, Almuzaini KS, Al-Refaee SA, Sulaiman MA, Dafterdar MY, Al-Ghamedi A, Al-Khuraiji KN. Aerobic and anaerobic power characteristics of Saudi elite soccer players. J Sports Med Phys Fitness 2001; 41; 1; 54-61.

32. Florida-James G, Reilly T. The physiological demands of Gaelic football. Br J Sports Med 1995; $29 ; 1 ; 41-45$.

33. Muñoz J, Huici M, Marcos E. Estudio de composición corporal y el somatotipo de deportistas sevillanos de alto rendimiento y comparación con la élite mundial. Arch Med Deporte 1986; 3; 11; 253 262. 